low-up $7 \%$ were cured, $54 \%$ improved, and $25 \%$ were unchanged. The remainder were worse or had died. However, $93 \%$ of them had persisting painful dysaesthesiae. At the time of development of the neurological picture there were associated systemic disturbances with raised blood pressure and white cell count in the peripheral blood, a raised erythrocyte sedimentation rate, and glycosuria. The aetiology of this condition is not known for certain, though the persistent painful dysaesthesiae have some resemblance to those seen after thalidomide intoxication. ${ }^{2}$

Epidemiological evidence has recently been advanced by T. Tsubaki and colleagues ${ }^{3}$ associating the syndrome with clioquinol therapy. This drug is generally available in the United Kingdom and many other parts of the world as EnteroVioform and is taken by travellers to prevent minor gastrointestinal upsets. It is for this reason that attention should be directed to a syndrome which until now has been rarely recognized outside Japan. Tsubaki and colleagues studied the records of 263 patients with various digestive disorders who received clioquinol therapy, comparing them with the records of 706 patients with similar disease who did not receive clioquinol. No neurological symptoms appeared in the latter cases, but $44(16.7 \%)$ cases of subacute myelo-opticoneuropathy appeared in the clioquinol group. Of those patients receiving therapy for more than 14 days 35\% developed the syndrome, while only $2.6 \%$ of those treated for 13 days or less were afflicted. They also found that 166 of 171 patients with the neurological syndrome whom they reviewed had received clioquinol therapy for the prodromal abdominal symptoms. On the basis of these findings they implicated clioquinol as the possible cause of the neurological disease, and the Japanese Ministry of Health and Welfare withdrew permission for the production and sale of clioquinol in Japan from September 1970.

Clioquinol had previously been thought to be remarkably safe for long-term administration. For instance, in the United States L. M. Gholz and W. L. Arons ${ }^{4}$ prescribed this drug in a dose of $750 \mathrm{mg}$ daily for four years to 4,000 institutionalized patients. They reported uniform success in preventing gastrointestinal disorders and extremely rare toxic sideeffects. They did, however, note that 20 patients developed "an unusual gait change". Eighteen of them recovered completely and 13 were later able to take the drug for long periods without recurrence of the disturbance of gait. The dosage given in the Japanese cases (0.6-1.6 g per day) was of the same order as used in this American study. Nevertheless over the years since the halogenated hydroxyquinolines were introduced a number of cases of optic atrophy and occasional damage to the spinal cord have been reported. ${ }^{5-7}$ Moreover Entero-Vioform itself was said to be cause of optic atrophy in two recently reported cases from Germany. ${ }^{8}$

W. T. Cooke and W. T. Smith ${ }^{9}$ reported degeneration of the spinal cord and peripheral nerves in patients with adult coeliac disease, though the cause of the degeneration was not discovered. It is therefore possible that the relationship to clioquinol therapy in the Japanese cases may be fortuitous. For instance, Tsubaki and colleagues' control group, who received no clioquinol therapy, may have differed either in type or in severity of abdominal disorders from those receiving the drug. Nevertheless the evidence these authors advance deserves careful attention. It is possible that the abdominal condition itself which developed in these Japanese patients was responsible for abnormal sensitivity to clioquinol, or that there is an underlying genetic predisposition to intoxication with this drug in the Japanese.
More reports may soon appear linking neurological diseases with clioquinol therapy. It is important that their significance should be assessed in relation to the very large quantity of clioquinol being taken throughout the world, and that attention should be given to the underlying abdominal condition for which the drug is given and to ethnic factors.

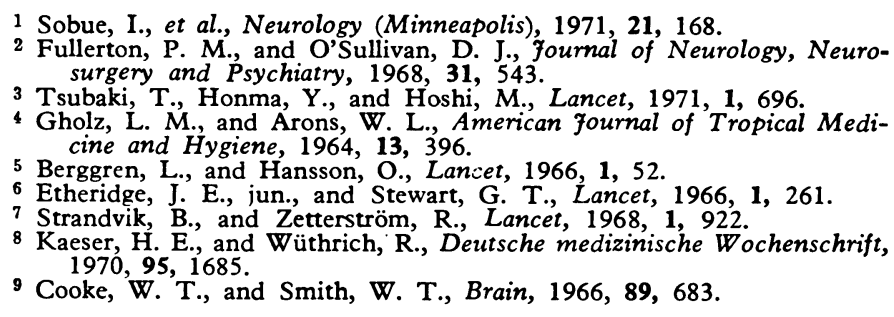

\section{Progress in Myeloma}

In recent years there has been considerable progress in our understanding of myeloma and in its treatment. Many aspects of the subject were discussed by leading authorities from all over the world at a "myeloma workshop" held recently in London, and shortened versions of most of their contributions are printed in this week's B.M.F. (page 319). All those attending the meeting agreed that it was outstanding of its kind, and its two scientific secretaries, Dr. D. A. G. Galton and Professor J. R. Hobbs, and the Leukaemia Research Fund, which sponsored it, are to be congratulated.

Myelomatosis is one of the few malignant diseases in which the cells regularly produce measurable amounts of metabolites that give some indication of the amount of tumour present. These metabolites are serum paraproteins and may be $\operatorname{IgA}, \operatorname{IgG}$, or $\operatorname{IgM}$ in type, and Hobbs has recently reviewed their significance in the disease. ${ }^{1}$ The studies reported at the workshop by J. L. Fahey and S. Salmon, together with previous ones by Hobbs and others, on the turnover rates of serum immunoglobulins indicate that most IgG and $\operatorname{IgA}$ myelomas may double their size in about 6 to 12 months and that as few as four doublings may occur from the time of early disease until death. If the growth of the tumour was constant and exponential, it could be calculated that the tumour must have started to grow some 15 to 20 years previously. Nevertheless, Salmon's measurements have shown that myeloma growth is not constant and that the rate of doubling becomes less as the number of tumour cells increases-as is the case with several other tumours in animals and man. His findings suggest that the early stages of tumour growth usually occur about five years before the disease is diagnosed.

Another topic discussed at the workshop was extramedullary plasmacytoma. In a review of 266 patients E. Wiltshaw showed that three-quarters of them had presented with a tumour of the upper respiratory tract and that the spread of the disease was much more like that of reticulum cell sarcoma than myelomatosis. The early invasion of lymph nodes was characteristic, and even when the disease became generalized the bone marrow was rarely involved. On the other hand, she emphasized that solitary myeloma of bone is merely an unusual form of myelomatosis and when disseminated is indistinguishable from the typical disease.

Another interesting discovery about myelomatosis is that in both experimental animals and in man the immunoglobulin produced by the malignant cells may have a specific affinity for a particular antigen. Thus the serum of one patient de- 
scribed by A. Videbaek contained a raised level of IgG associated with a high titre of antistaphylolysin, while in another with an IgA type of paraprotein there was high activity against the streptococcal $O$ fraction. It was suggested several years ago that a single antigen could lead to the formation of IgM antibody and later to IgG antibody by the same cell, ${ }^{2-4}$ and work by $\mathrm{H}$. H. Fudenberg and his colleagues has provided additional evidence for this concept. On the other hand, the recently recognized association between myelomatosis and the subsequent development of acute myelomonoblastic leukaemia is still unexplained. ${ }^{5}$ Even more perplexing is the finding in one patient described by Videbaek of a bone marrow infiltrated by apparent myeloblasts which contained IgG, as did the myeloma cells. This illustrates how difficult it may be to make a diagnosis of either of these conditions on purely morphological grounds.

Even if chemotherapy with modern drugs has been responsible for producing leukaemia in some patients with myeloma, E. F. Osserman was convinced that this was a "reasonable price" to pay for the benefits of treatment. Certainly the introduction of treatment by melphalan and cyclophosphamide has considerably increased the survival rates of patients with myelomatosis. This improvement could be explained on the grounds that the drugs inhibited only one to two doublings, and J. D. Bergsagel suggested that a similar effect might be produced by total body irradiation. The Medical Research Council myeloma trial, comparing the effects of continuous melphalan and cyclophosphamide in 276 patients, ${ }^{6}$ showed no significant difference between the survival of patients treated with either drug. Nevertheless, the trial provided valuable information about the factors which influence prognosis. A low level of serum albumin, heavy Bence Jones proteinuria, and a raised concentration of blood urea were all found to indicate a poor prognosis. Thus over half the patients who presented with a blood urea of more than $80 \mathrm{mg} / 100 \mathrm{ml}$ were dead within two months of admission. Similarly a rapid response to chemotherapy was associated with a short survival, possibly because in these patients the myeloma cells are multiplying particularly rapidly.

' Hobbs, J. R., British Medical Fournal, 1971, 2, 67.

Bauer, D. C., and Stavitsky, A. B., Proceedings of the National Academy of Sciences of the United States of America, 1961, 47, 1667.

s Uhr, J. W., and Finkelstein, M. S., Fournal of Experimental Medicine, $1963,117,457$.

- Nossal, G. J. V., Szenberg, A., Ada, G. L., and Austin, C. M., fournal of Experimental Medicine, 1965, 119, 485.

s British Medical fournal, 1971, 1, 568.

- Medical Research Council, British Medical fournal, 1971, 1, 640.

\section{New Research on Cannabis}

A recent bibliography ${ }^{1}$ of writings on cannabis listed 1,656 items. Many of the papers bear titles which are variations on "The Menace of Marihuana" or the polar theme "In Defence of Pot". There is a repetition of familiar arguments and much evidence of that special kind of moral fervour which springs from the conjunction of an abundance of passion and a scantiness of facts.

But in recent years a number of reports have been published which between them begin to lay a groundwork of solid understanding about the nature of the active constituents of cannabis and their psychopharmacological action. In the latest such report J. E. Manno and his colleagues ${ }^{4}$ give a specially interesting example of the manner in which new knowledge of the pharmacology of cannabis allows precise measurement of the dose of the active constituent and hence provides a basis for exact laboratory experiment. These workers assayed the quantity of delta-9-tetrahydrocannabinol (THC) in a batch of Asian marihuana, and with the aid of a smoking machine calculated that approximately $50 \%$ of the active constituent is destroyed by heat in the process of smoking. They also made a small correction for the quantity left over in the butt-end. They were able then to calculate with a fair degree of certainty that a calibrated cigarette they prepared would deliver THC in a dose of $5 \mathrm{mg}$. A placebo cigarette was made up by extracting the cannabinols from the marihuana. When in a double-blind cross-over trial the effects of cannabis and placebo were compared, the eight persons tested showed clear decrement in performance on motor and mental tasks after the cannabis smoking. The test of motor function entailed a version of the "pursuit task", which is a familiar tool of psychological laboratories: the person controlled an oscilloscope dot and had to follow the tracing of a pattern across the tube's face. The mental testing included a number of simple arithmetical and reading tasks performed under the stressed condition of the subject listening to his own voice delayed through headphones.

The question arises how such laboratory tests may be related to the circumstances of daily life. This is a question, not a criticism, for the setting up of even the most artificial situation which allows precise measurement of a drug's effect provides a research tool of value. But anyone reading this report by Manno and his colleagues must be set wondering how cannabis may effect driving skills and other ordinary social tasks with a low margin of safety.

Work from another American laboratory bears on these matters. A. T. Weil and N. E. Zinberg ${ }^{5}$ investigated in an elegant series of experiments the impairment by cannabis of ultra-short-term memory and speculated on the impact such impairment might have on driving. They quote the following remarks of a cannabis-smoking doctor: "I often drive my automobile when I'm high on marihuana and have never had any actual problems doing so. I'll come to a stop light and have a moment of panic because I can't remember whether or not I've put my foot on the brake. Of course, when I look down, it's there, but in the second or two afterwards I can't remember having done it". When the influence of cannabis and of alcohol on simulated driving performance was investigated, ${ }^{6}$ the influence of cannabis in the particular dose given appeared to be minimal. The people tested tended to glance at the speedometer rather less often.

This new wave of refined laboratory research coming from America takes matters much further than was possible before the dosage of cannabis could be measured precisely. Older work $^{7}$ had in essence simply shown that where a person is acutely intoxicated with cannabis he experiences an overall and non-specific reduction in motor and intellectual functioning. Better experimental design will also come from taking into account the person's previous experience with cannabis, because naive and practised smokers react differently. ${ }^{8}$ Though laboratory work in the next few years will provide new and important facts, parallel research in other disciplines is also needed. Study of the social aspects of drug taking ${ }^{9}$ and of the determinants of society's response to $\mathrm{it}^{10}$ will become more rational when exact observations replace old myths." 